\title{
Corrigendum Regarding the Article by Song et al. Entitled 'Renal Replacement Therapy for Prevention of Contrast-Induced Acute Kidney Injury: A Meta-Analysis of Randomized Controlled Trials'
}

\author{
Dinna N. Cruz ${ }^{a}$ Mark A. Perazellab \\ aDepartment of Nephrology, San Bortolo Hospital and International Renal Research Institute Vicenza, Vicenza, Italy; \\ bSection of Nephrology, Department of Internal Medicine, Yale University School of Medicine, New Haven, Conn., USA
}

To the Editor

We read with interest the meta-analysis by Song et al. [1] on renal replacement therapy (RRT) for the prevention of contrast-induced acute kidney injury (CI$\mathrm{AKI}$ ). In principle we agree that whatever benefit prophylactic RRT may offer, this is likely limited to a high-risk population with a high pretest probability of CI-AKI, including those with severe chronic kidney disease (CKD). While this seems quite intuitive, we might argue that this remains speculative and not borne out by the metaanalysis.

In table 1 , the 9 studies are classified by CKD stage; 5/9 directly reported a mean estimated glomerular filtration rate (eGFR) or creatinine clearance $(\mathrm{CrCl})$. Among these, the mean $\mathrm{CrCl}$ in Frank et al. [2] was $17.4-19.4 \mathrm{ml} / \mathrm{min}$; such patients would be classified as stage 4 rather than stage 3 as erroneously indicated in table 1 .

The remaining 4/9 studies reported only mean serum creatinine ( $\mathrm{sCr}$ ) values, mean age and genders of both study arms. We would presume therefore that CKD staging was performed by approximating eGFR based on these available data. Since all 4 studies were conducted in Europe, the subjects are likely to be predominantly Caucasian. If so, the lowest possible approximate eGFR would be obtained by using the higher mean age and $\mathrm{sCr}$, selecting 'female' gender and sCr traceable by iso- tope dilution mass spectrometry. The highest possible eGFR would be obtained by using the lower mean age and sCr, selecting 'male' gender and sCr non-traceable by isotope dilution mass spectrometry. The range of imputed eGFRs would therefore be 1319 [3], 11-26 [4], 16-28 [5] and 19-32 [6] ml/ $\min / 1.73 \mathrm{~m}^{2}$. Therefore only the study by Lehnert et al. [6] could conceivably be classified as CKD stage 3-4, while the others are stage 4 . Song et al. inappropriately classified these studies as stage 3 .

Furthermore, in figure 3 of Song et al. [1], they indicate the number of patients who develop CI-AKI for each study.

The primary end point in the study of Lee et al. [7] was the change in $\mathrm{CrCl}$ between baseline and the fourth day; the incidence of CI-AKI was not among their reported end points. What they did report was that 18 patients in the control group and 2 in the RRT group had permanent renal damage with an increase in $\mathrm{sCr}>1 \mathrm{mg} /$ $\mathrm{dl}$ or required permanent dialysis after discharge. Clearly this is not an end point that can be combined with the others in figure 3 .

If the misclassification of CKD stages and the aberrant data abstraction for the study of Lee et al. are taken into consideration, the meta-analysis no longer demonstrates a differential effect between CKD stage 3 and CKD stage $4-5$.

\section{References}

${ }_{1}$ Song K, Jiang S, Shi Y, Shen H, Shi X, Jing D: Renal replacement therapy for prevention of contrast-induced acute kidney injury: a meta-analysis of randomized controlled trials. Am J Nephrol 2010;32:497-504.

2 Frank H, Werner D, Lorusso V, et al: Simultaneous hemodialysis during coronary angiography fails to prevent radiocontrast-induced nephropathy in chronic renal failure. Clin Nephrol 2003;60:176-182.

-3 Vogt B, Ferrari P, Schonholzer C, et al: Prophylactic hemodialysis after radiocontrast media in patients with renal insufficiency is potentially harmful. Am J Med 2001;111: 692-698.

-4 Sterner G, Frennby B, Kurkus J, Nyman U: Does post-angiographic hemodialysis reduce the risk of contrast-medium nephropathy? Scand J Urol Nephrol 2000;34:323-326.

-5 Berger ED, Bader BD, Bosker J, Risler T, Erley CM: Contrast media-induced kidney failure cannot be prevented by hemodialysis (in German). Dtsch Med Wochenschr 2001;126: 162-166.

-6 Lehnert T, Keller E, Gondolf K, Schaffner T, Pavenstadt H, Schollmeyer P: Effect of haemodialysis after contrast medium administration in patients with renal insufficiency. Nephrol Dial Transplant 1998;13:358-362.

7 Lee PT, Chou KJ, Liu CP, et al: Renal protection for coronary angiography in advanced renal failure patients by prophylactic hemodialysis. A randomized controlled trial. J Am Coll Cardiol 2007;50:1015-1020.

\section{KARGER}

Fax +41613061234 E-Mail karger@karger.ch www.karger.com
Dinna N. Cruz, MD

Department of Nephrology

San Bortolo Hospital and International Renal Research

Institute Vicenza, Viale Rodolfi 37, IT-36100 Vicenza (Italy)

E-Maildinnacruzmd@yahoo.com 


\section{Reply}

\section{Kai Song}

Department of Nephrology, Second Affiliated Hospital of Soochow University, Suzhou, China

Dear Editor

We are terribly sorry for answering you so late. We have been trying to get in touch with Dr. Po-Tsang Lee and Dr. Hua-Chang Fang for the original data of their trial in the last week, but unfortunately we failed to get any reply.

Cruz and Perazella are right that we made mistakes on the baseline CKD staging and we are terribly sorry about that. Except for the study of Lehnert et al., the CKD stage of the study should be classified as CKD stage 4, rather than stage 3. However, if one scrutinized the baseline CKD stage, one may find that the corresponding GFR varied dramatically from 11 to $42 \mathrm{ml} /$ min, no matter for the race difference. Therefore, we still believe that the population included in those studies should not be viewed as one unified group. The difference of baseline GFR may be the source of the heterogeneity in our meta-analysis and that of Cruz and Perazella which can be confirmed by the results of meta-regression.

We did input the wrong data of the primary end point and that is why we kept asking Lee to give us the original data of the incidence of contrast-induced nephropathy. Lee reported the incidences of permanent renal damage in the control and RRT group. We premise that those patients with the $\mathrm{sCr}$ increased over $1 \mathrm{mg} / \mathrm{dl}$ developed contrast-induced nephropathy with Acute Kidney Injury Network criteria in which AKI occurs when $\mathrm{sCr}>0.3 \mathrm{mg} / \mathrm{dl}$ from baseline.

Lee's study enlightened us to perform our meta-analysis. Lee's study is important to us as this is the only one which substantiated the effectiveness of hemodialy- sis in the prevention of contrast-induced nephropathy in patients with severely impaired kidney function. As a result, we still believe that Lee's study should be included for the meta-analysis. We still hope that Lee could give us his original data so that we can reanalyze the data. More trials are still needed to substantiate the effectiveness of hemodialysis in patients with more advanced kidney function.

We thought that we might have found out why hemodialysis is ineffective in the prevention of contrast-induced nephropathy while contrast could be easily eliminated by this therapy. But now we realize that there is still a long way to go before a sound conclusion can be made, and some of our conclusions might be wrong. We apologize for all the trouble to you and your journal. 